\title{
Molecular Dynamics Study of Tryptophylglycine: A Dipeptide Nanotube with Confined Water
}

\author{
Yuansheng Pan, ${ }^{* \dagger}$ Henrik Birkedal, ${ }^{\dagger, *}$ Philip Pattison, ${ }^{\dagger, \S}$ David Brown,,$\|$ and Gervais Chapuis ${ }^{\dagger}$ \\ Laboratoire de Cristallographie, Ecole Polytechnique Fédérale de Lausanne, BSP, \\ 1015 Lausanne, Switzerland, Swiss-Norwegian Beam Line, SNBL/ESRF, P.O. Box 220, \\ F-38043 Grenoble, France, and Laboratoire des Matériaux Organiques à Propriétés Spécifiques, \\ UMR CNRS 5041, Université de Savoie, 73376 Le Bourget du Lac, France
}

Received: October 24, 2003; In Final Form: March 2, 2004

\begin{abstract}
To investigate the mechanism of structural changes of a peptide nanotube and water confined inside the channel, the helical peptide tryptophylglycine monohydrate $\left(\mathrm{WG} \cdot \mathrm{H}_{2} \mathrm{O}\right)$ was studied by molecular dynamics (MD) simulations using the three-dimension parallel MD program ddgmq (software package) and a consistent force field. Simulations were performed on both the water-containing system and a model system without water molecules. The details of the structural behavior with temperature are investigated for the entire simulated temperature range. Phase transitions were obtained at 115, 245, 270, 310, and $385 \mathrm{~K}$, due to the contributions of both the peptide and the confined water subsystems. The crystalline, amorphous, liquidlike, liquid, and superheated phases of water were observed in the temperature ranges $40-115,115-245,245-310,310-$ 385 , and $>385 \mathrm{~K}$, respectively. At $300 \mathrm{~K}$, the diffusion constant of the confined water is $0.46 \times 10^{-5} \mathrm{~cm}^{2}$ $\mathrm{s}^{-1}$, a value comparable to that of other peptide nanotubes. The empty peptide system melts at $440 \mathrm{~K}$. Mechanisms of the negative thermal expansion (NTE) along the tube axis were investigated for different temperature ranges. The contraction of the crystalline water (or amorphous water) draws also the tube walls in and leads to NTE below $245 \mathrm{~K}$. The other NTEs appear to be connected to the collapse of the ice network or the solid peptide network between $245 \mathrm{~K}$ and room temperature or from 310 to $440 \mathrm{~K}$, respectively.
\end{abstract}

\section{Introduction}

Following the discoveries of self-assembling peptide systems, ${ }^{1,2}$ synthetic and natural supramolecular tubular assemblies have become of great interest owing to their potential use as cross-membrane conduits, ${ }^{3,4}$ as membrane piercing antibacterials, ${ }^{5}$ and, in the case of synthetic systems, as model systems for natural tubular systems such as porins and ion-channel proteins. ${ }^{4,6}$ In 1993, Ghadiri and co-workers ${ }^{1}$ demonstrated that nanotubes can be self-assembled by a process based on $\beta$-sheetlike interactions between macrocyclic D,L-peptides. Subsequent work by Ghadiri and several others ${ }^{4,7-11}$ revealed other examples of polypeptide architecture that could lead to nanotube formation through vertical stacking of cyclic peptides. In the chemical literature, these peptidic systems have received significant interest because of their properties, but the alternative use of carbon nanotubes ${ }^{12}$ has also been the focus of strong efforts.

It was recently found by Görbitz that certain linear L,Ldipeptides also form peptide nanotubes. ${ }^{9}$ In L-Leu-L-Leu (LL), L-Leu-L-Phe (LF), and L-Phe-L-Leu (FL), four dipeptide molecules form the outskirt of a hydrophilic region, which contains water molecules in the core channel. The four dipeptide molecules display a pseudotetragonal symmetry, with a waterfilled channel of $10 \AA$ diameter. In a similar way, the crystal

* Corresponding author. Telephone: +41-21-693 0638. Fax: +41-21693 0504. E-mail: Yuansheng.Pan@epfl.ch.

† Ecole Polytechnique Fédérale de Lausanne.

$\doteqdot$ Present address: Department of Chemistry, University of Aarhus, Langelandsgade 140, DK-8000 Aarhus C, Denmark.

$\S$ Swiss-Norwegian Beam Line.

" Université de Savoie.

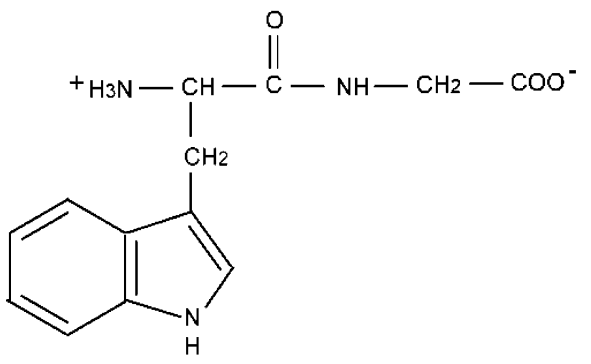

Figure 1. Tryptophylglycine.

structure of six dipeptide molecules of L-Phe-L-Phe (FF) create a somewhat larger channel.

Tryptophylglycine monohydrate $\left(\mathrm{WG} \cdot \mathrm{H}_{2} \mathrm{O} \text {, Figure } 1\right)^{10,11}$ is another example of a dipeptide that forms an extended, onedimensional, water-filled tubular structure. This system displays several unusual features, one in particular being uniaxial negative thermal expansion along the tube axis. ${ }^{11}$ Here, the symmetry is truly tetragonal, with space group symmetry $P 4_{1}$. The tube axis is parallel to the tetragonal $c$-axis. Compared to the rather confined volumes in the channels of LL and LF with dimensions of $2.5 \times 6.0 \AA$ and FL with $4.0 \times 6.0 \AA$, the internal cross section of the WG nanotube, $8.3 \times 8.3 \AA$, is relatively large. WG is a dipeptide expressed in the human hypophysis. The activity of this compound is not yet known. It was located as a fluorescent hypophyseal extract from adult human brains. ${ }^{13}$ Vellucci and Webster found a sedative effect in mice, evidenced by a decreased locomotor and exploratory activity. ${ }^{14}$ They further found that WG also reduces rectal temperature, decreases the convulsive action of the drug leptazol, and increases the antileptazol effect of diazepam in mice. 


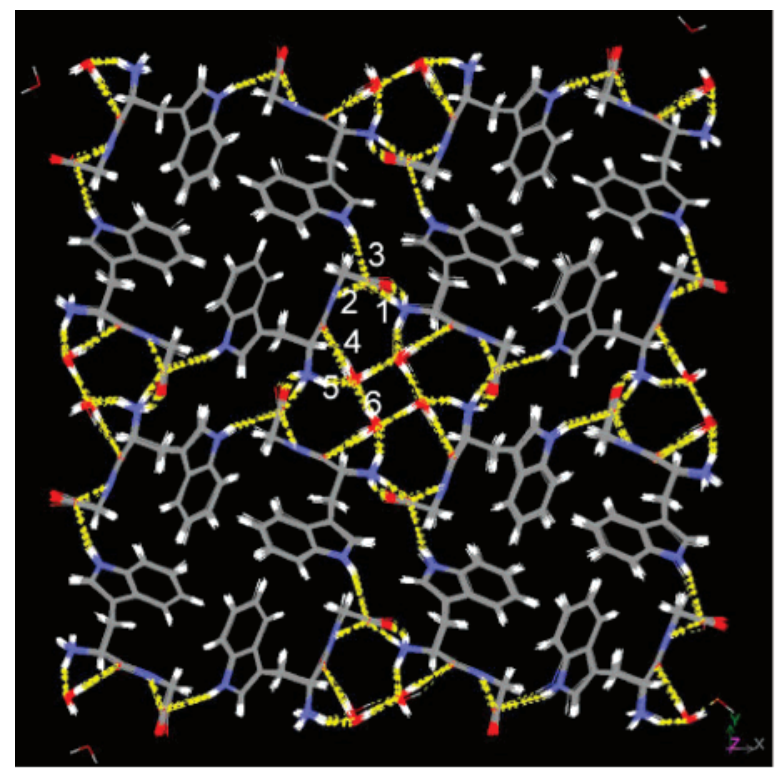

$\mathbf{a}$

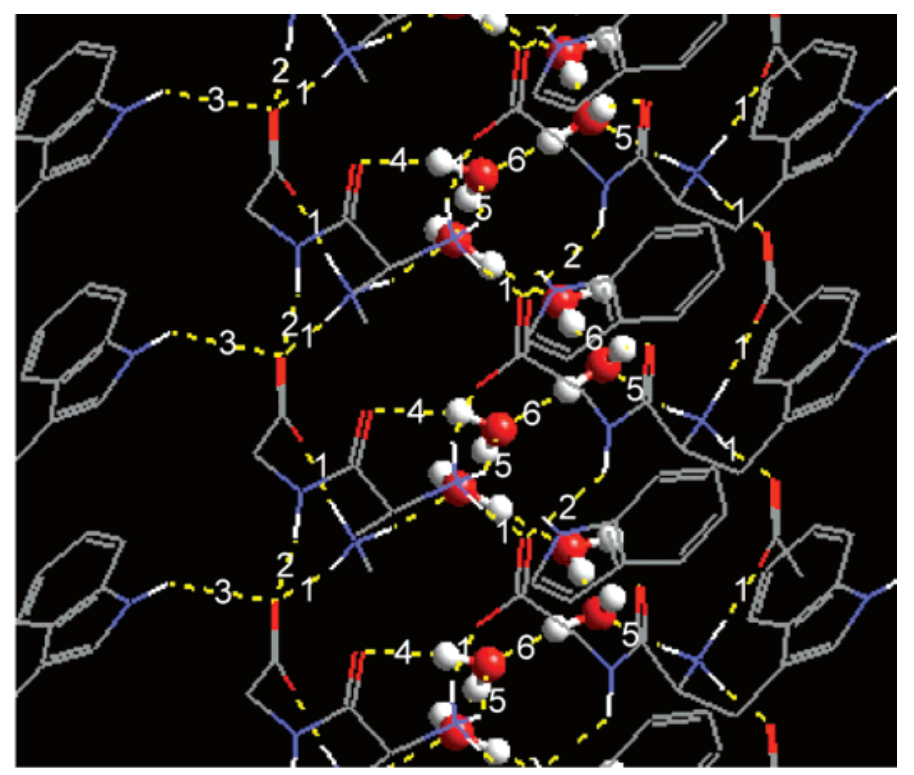

b

Figure 2. Simulated crystal structure of $\mathrm{WG} \cdot \mathrm{H}_{2} \mathrm{O}$ at $40 \mathrm{~K}$ (one time step shown): (a) $x y$ plane and (b) $x z$ plane. To clearly show the hydrogenbonding scheme, some dipeptide molecules were removed from the figure. Four columns of WG molecules connected by hydrogen bonds in a helical structure form a nanotube; a water helix is confined within the nanotube. The connection between different nanotubes is via the hydrogen bond " 3 ". Along the $c$ direction, the framework of the tube is mainly supported by hydrogen bonding to the terminal carboxylate group.

Water confined inside nanotubes is also attracting new interest. Water already exhibits a rich spectrum of phase behavior in the bulk. ${ }^{15-19}$ Investigations of water confined inside a nanotube found different crystalline structures and phase transitions compared to the bulk case.$^{20-26}$ Molecular dynamics simulations have been used to study a few nanotubular systems. For a cyclic octapeptide system with internal dimensions similar to those of $\mathrm{WG} \cdot \mathrm{H}_{2} \mathrm{O}$, MD simulations of an isolated tube of 10 such molecules in water revealed the dynamics of the water molecules inside the channels. ${ }^{20}$ In another simulation, Hummer et al. ${ }^{21}$ investigated the channeling of water molecules through a carbon nanotube of $8.1 \AA$ diameter. In this system, the water molecules are only subject to van der Waals interactions with the carbon atoms of the nanotube (unlike the hydrogen bonds linking water and peptide molecules as in WG, Figure 2). The hydrophobic carbon nanotubes were spontaneously filled with a one-dimensionally ordered chain of water molecules. Koga et al. ${ }^{24}$ reported the existence of new ice phases and confined phase transitions in water encapsulated in carbon nanotubes.

We have recently used molecular dynamics simulation to model several crystal structures. By introducing a compensating pressure tensor field, it is possible to reproduce the lattice constants and angles with a precision comparable to the experimental measurements. ${ }^{27}$ This technique was used successfully for the simulation of the commensurate-incommensurate phase transition in the hexamethylenetetramine suberate system. ${ }^{28}$

To gain insight into the origin of any phase transition and negative thermal expansion along tube axis, we decided to apply MD techniques to $\mathrm{WG} \cdot \mathrm{H}_{2} \mathrm{O}$.

\section{Methods and Model}

2.1. Program. Molecular dynamics simulations were performed using the parallel MD program ddgmq, which was principally designed for the simulation of dense materials in three dimensions (3D) with periodic boundary conditions. ${ }^{29}$ The time evolution of a set of interacting atoms is obtained by integrating their Newton's equations of motion using the leapfrog-Verlet algorithm, with a time step of $1.0 \mathrm{fs}$. In $d d g m q$,
TABLE 1: Lattice Constants and Angles at 120 and $295 \mathrm{~K}^{a}$

\begin{tabular}{lllcclcl}
\hline$T / \mathrm{K}$ & system & \multicolumn{1}{c}{$a / \AA$} & \multicolumn{1}{c}{$b / \AA$} & $c / \AA$ & $\alpha / \mathrm{deg}$ & $\beta / \mathrm{deg}$ & $\gamma / \mathrm{deg}$ \\
\hline 120 & experiment & 16.012 & 16.012 & 5.207 & 90.00 & 90.00 & 90.00 \\
& MD, hyd & 16.0134 & 16.0138 & 5.2064 & 89.996 & 89.998 & 90.000 \\
& MD, anhyd & 16.287 & 16.285 & 5.1274 & 89.994 & 90.004 & 90.017 \\
295 & experiment & 16.164 & 16.164 & 5.199 & 90.00 & 90.00 & 90.00 \\
& MD, hyd & 16.2632 & 16.2763 & 5.1572 & 89.971 & 89.988 & 90.013 \\
& MD, anhyd & 16.3955 & 16.4016 & 5.1433 & 90.000 & 89.994 & 90.003
\end{tabular}

${ }^{a}$ Experimental data and the simulations with (hyd) and without (anhyd) water are indicated. The anhydrous result cannot be compared directly to the experimental data because the two systems are inherently different.

molecules are modeled as a number of atoms connected by a network of bonds. All atoms interact with all other "nonbonded" atoms through pair potentials. In the present case, the nonbonded pair potentials are the Lennard-Jones (LJ) 9-6 and the Coulombic potentials. Bonded potentials include terms to describe bond stretching, bond angle bending, torsional rotations, and out-of-plane deformations. All the high-frequency modes associated with hydrogen atoms in $\mathrm{CH}_{2}$ groups were removed using special constraints. ${ }^{30}$ The truncation range of nonbonded pair potentials is $9 \AA$.

2.2. Starting Model and Force Field. All potential parameters are taken from a second-generation consistent force field (CFF). ${ }^{31,32}$ All charges were calculated by the equilibration approach included in the Cerius 2 package, ${ }^{33,34}$ which estimates the appropriate values according to the molecular geometry and the atomic electronegativities. This approach allows the charges to respond to changes in the environment. Charges were calculated from the starting model and thereafter kept fixed during the MD runs. The starting model was taken from the $120 \mathrm{~K}$ single-crystal structure of Birkedal et al. ${ }^{11}$ The coordinate system was chosen with $Z$ parallel to the crystallographic $c$-axis. Lattice constants and angles of the starting structure are indicated in Table 1. The simulation system contains $2 \times 2 \times 20$ unit cells with 11840 atoms $(32 \times 32 \times 104 \AA)$. The Ewald method is applied to handle the Coulombic potential. ${ }^{35,36}$ In $d d g m q$, three parameters control the convergence of the Ewald summation, 
$\alpha, K_{\mathrm{MAX}}$, and $R_{\mathrm{MAX}}{ }^{27}$ They were given values of $0.28 \AA^{-1}, 8.0$ $\AA$, and $9.0 \AA$ A, respectively.

2.3. MD Procedures. Prior to varying the temperature, the structure was equilibrated at $120 \mathrm{~K}$ at constant $V$. For a perfect force-field and a large enough sample, the result of this procedure should coincide with the experimental results. However, due primarily to the deficiencies in the generic force field, the experimental lattice parameters are in general not accurately reproduced. To correct this effect, a compensating pressure tensor was determined at $120 \mathrm{~K}$ and applied throughout all further simulations. In an initial 550 ps NVT (constant number of particles, volume, and temperature) run at $120 \mathrm{~K}$, the starting system was brought to equilibrium while the lattice constants were kept unchanged. This allowed us to determine the pressure tensor, which is diagonal owing to tetragonal symmetry $P_{x x}=$ $P_{y y}=3121$ bar, $P_{z z}=7441$ bar, $P_{x y}=P_{x z}=P_{y z}=0$. In the next step, the system was equilibrated at the same temperature under NPT conditions (constant number of particles, pressure, and temperature) with the compensating pressure tensor applied. The lattice parameters obtained at equilibrium are shown in Table 1 . The validity of the compensating pressure tensor at other temperatures can be checked by comparison of the simulation results and experimental measurements.

2.4. MD Runs. Two independent peptide systems were studied: one containing water molecules and one without. The former is equivalent to the experimental results at low temperature, while the latter was studied in order to gain insight into the role of water with respect to the negative thermal expansion and to investigate the stability of the water-free peptide tube in the crystalline environment. The same pressure tensor was used in both simulations. Lattice constants and angles listed in Table 1 result from the system after reaching equilibrium at $120 \mathrm{~K}$.

The first simulation was carried out with water inside the nanotube. At $120 \mathrm{~K}$, the equilibrated system was both heated at a rate of $1 \mathrm{~K} / \mathrm{ps}$ to $450 \mathrm{~K}$ and cooled at $-1 \mathrm{~K} / \mathrm{ps}$ to $40 \mathrm{~K}$ by NPT dynamics including the compensating pressure tensor. TGA experimental observations indicated that the confined water is lost in the temperature range from 317 to about $420 \mathrm{~K}$. However, our MD simulations were carried out within 3-D periodic boundary conditions, which do not allow the water to leave the system. Therefore, this first simulation focus mainly on the temperature range before the water is lost.

To simulate the system after the water has evaporated, another simulation was performed by simply removing the water from the channel. The starting structure was the same as the first simulation, except for the absence of the water molecules. All charges were calculated from this starting model by the equilibration approach. The system was equilibrated under NPT conditions with the same compensating pressure tensor applied. The equilibrated system was then heated at a rate of $1 \mathrm{~K} / \mathrm{ps}$ from 120 to $520 \mathrm{~K}$.

\section{Results}

3.1. Simulation with Water-Containing Channels. We first describe the system containing water in which we observe several phase transitions.

3.1.1. Lattice Constants and Phase Transitions. The lattice constants of both simulated systems are presented in Figure 3. A change of slope of the lattice constants at a specific temperature indicates a phase transition. Such slope changes can be observed at about $115,245,270,310$, and $385 \mathrm{~K}$ for the water-containing channel represented in Figure 3a,b. In this simulation, the lattice angles did not deviate from $90^{\circ}$ up to $385 \mathrm{~K}$. A phase transition occurring in the peptide or the water

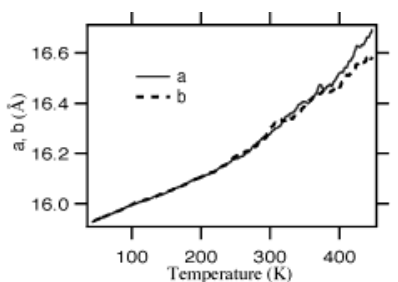

a

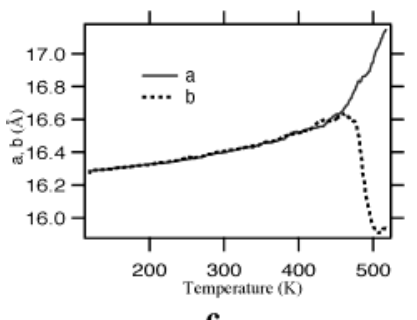

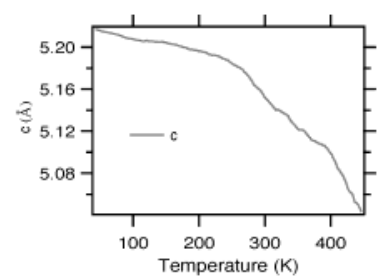

b

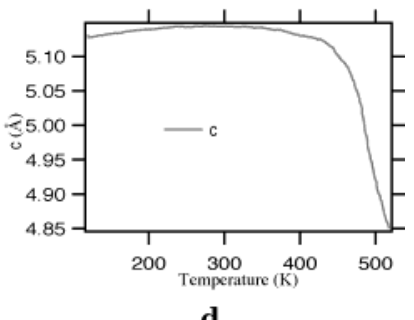

d
Figure 3. Simulated lattice constants of $\mathrm{WG} \cdot \mathrm{H}_{2} \mathrm{O}$. Parts a and $\mathrm{b}$ display the results for the water-containing system, while parts $\mathrm{c}$ and $\mathrm{d}$ refer to the water-free system. Blue lines indicate the $a$-axis, dashed black lines the $b$-axis, and red lines the $c$-axis. For the water-filled channels, the changes of slope in a and $\mathrm{b}$ indicate phase transitions near 115, $245,270,310$, and $385 \mathrm{~K}$. In the water-free system, the onset of differences between the $a$ - and $b$-axes at $\sim 450 \mathrm{~K}$ indicates of loss of symmetry.

subsystem can cause changes in lattice parameters. Both subsystems will be studied to elucidate the mechanism of these transitions. Table 1 compares the lattice constants with the experimental results at $295 \mathrm{~K}$. The largest deviation of the lattice constants is $0.8 \%$ and the angles agree well with experiment. This indicates that the estimation of the thermal expansion is fair. Most significantly, the simulations reproduce the observed negative thermal expansion ${ }^{11}$ along the tube axis (Figure $3 b$ ).

Figure $4 \mathrm{a}$ shows the mean square displacements (MSDs) of all atoms, oxygen atoms of water, and peptide nitrogen atoms of the peptide. The $\mathrm{N}$ atoms display very small MSDs, which increase uniformally with temperature over the entire simulation range, except for a change of slope at $385 \mathrm{~K}$. This is in accordance with the corresponding increase of the thermal (kinetic) energy of the system. The MSD of all the atoms shows slope changes at $115,245,270,310$, and $385 \mathrm{~K}$. With contributions from both entities, these values are intermediary. The MSD of the water's oxygen atoms displays a drastically different behavior with strong slope changes at these temperatures, indicating an increase of the water mobility, which can be interpreted as phase transitions. Note that after the transitions, all the water is still confined within the nanotubes, owing to the periodic boundary conditions imposed on our simulation. Figure $4 \mathrm{~b}$ indicates changes of intermolecular van der Waals energy of the system in both simulations. For the watercontaining channel, the phase transitions at 245 and $270 \mathrm{~K}$ are accompanied with a drop of $3.3 \mathrm{~kJ} \mathrm{~mol}^{-1}$ in the van der Waals energy occurring between 245 and $310 \mathrm{~K}$. This energy drop is comparable to that $\left(3.7 \mathrm{~kJ} \mathrm{~mol}^{-1)}\right)$ in confined TIP4P water between two parallel walls ${ }^{38}$ and that $\left(7.0 \mathrm{~kJ} \mathrm{~mol}^{-1}\right)$ at the freezing transition of TIP4P bulk water. ${ }^{39}$ In this temperature range, this is due to the breaking of hydrogen bonds between water molecule and tube wall and between water molecules. The details of the transition mechanisms are illustrated in the next section.

3.1.2. Mechanisms of Phase Transitions. Phase transitions usually correspond to changes in the structure. Their mechanism can be investigated by studying the details of the structures revealed by the simulations. Seven different types of hydrogen 


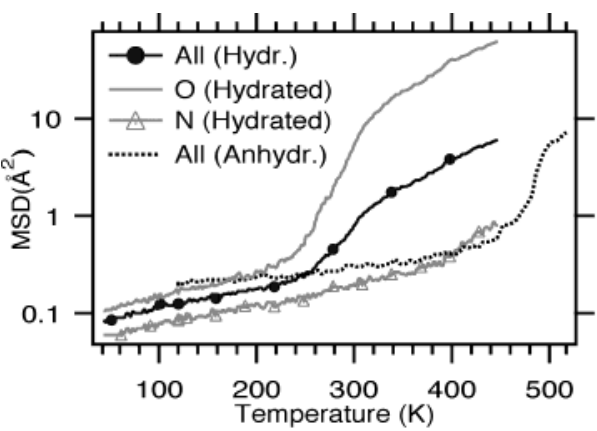

a

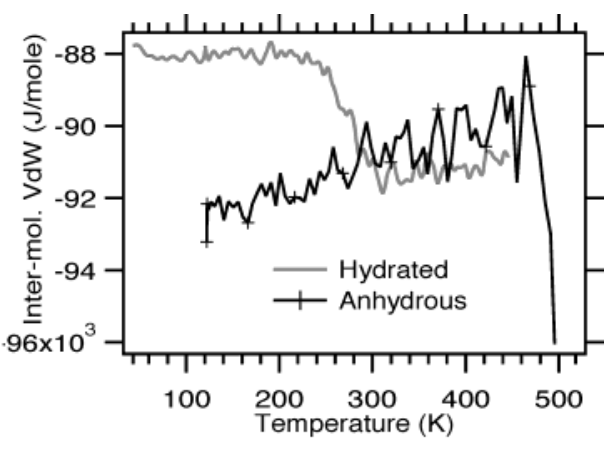

b

Figure 4. (a) Mean square displacements (MSDs) of all atoms, of the $\mathrm{O}$ atom of the water molecules, and of the $\mathrm{N}$ atom of the peptide molecules in the hydrated system and of all atoms in the anhydrous system. (b) Intermolecular van der Waals energy of both simulations.

TABLE 2: Hydrogen-Bond Types and Connecting Atoms in the Water-Containing System

\begin{tabular}{cl}
\hline HB type & \multicolumn{1}{c}{ connecting atoms } \\
\hline 1 & $\mathrm{NH}_{3} \cdots \mathrm{OC}(1), \mathrm{NH}_{3} \cdots \mathrm{OC}(2)$ \\
2 & amide nitrogen $\mathrm{NH} \cdots \mathrm{OC}$ \\
3 & indole nitrogen $\mathrm{NH} \cdots \mathrm{OC}$ \\
4 & water $\mathrm{OH} \cdots \mathrm{OC}$ \\
5 & $\mathrm{NH}_{3} \cdots \mathrm{OH}$ water \\
6 & water $\mathrm{OH} \cdots \mathrm{OH}$ water
\end{tabular}

bonds (HBs) can be identified in the optimized low-temperature $(40 \mathrm{~K})$ structure (see Table 2 and Figure 2). Four of them [two $\mathrm{NH}_{3} \cdots$ OC bonds ( 1 in Figure 2 ), one $\mathrm{NH} \cdot \cdot \mathrm{OC}$ bond involving the amide nitrogen ( 2 in Figure 2 ), and an additional $\mathrm{NH} \cdots \mathrm{OC}$ bond with the indole nitrogen (3 in Figure 2)] are connected to the neighboring WG molecules. Two HBs [one $\mathrm{OH} \cdots \mathrm{OC}$ (4 in Figure 2) bond and one $\mathrm{NH}_{3} \cdots \mathrm{OH}$ bond (5 in Figure 2)] link WG and a water molecule, and one bond connects water molecules (6 in Figure 2).

Figure 2 shows that the peptide framework is maintained by hydrogen bonds linking the terminal $\mathrm{COO}$ group of the dipeptide along $c$ and that the peptide channels are connected by hydrogen bonds of type 3 (HB 3 ) in the $x y$ ( $a$ and $b$ ) direction. One helix of water molecules and the surrounding peptide framework form a channel interconnected by hydrogen bonds of types 4 and 5 (HB 4 and HB 5). It is thus reasonable to expect that the changes in lattice constants at the phase transitions are linked to changes in the terminal OCCN torsion angles of $\mathrm{WG}$ and the number of hydrogen bonds of type 3 . The temperature evolution of these parameters is shown in Figure 5. Figure 5a shows that the changes of torsion angles OCCN with temperature present the same features as the behavior of the lattice constant $c$. The temperature dependence of the torsion angles changes slopes at approximately the same phase-transition temperatures found for the lattice constants. As illustrated in Figure 5b, the number of hydrogen bonds of type HB 3 smoothly decreases with temperature following the changes of the lattice constants $a$ and $b$ up to about $200 \mathrm{~K}$, above which the number of HB 3's stabilizes. The number of hydrogen bonds of this type decreases by $50 \%$ from 40 to $200 \mathrm{~K}$. This causes the tryptophan indole rings, connected by $\mathrm{HB} 3$, to release their hydrogen bonds and to extend their distribution into a larger volume. The system increases its dynamical disorder from 40 to $200 \mathrm{~K}$.

A more detailed analysis of the structure reveals the origin of the phase transitions. The OCCN torsion angles present the combined effect of changes of HB types 1, 2, 4, and 5. Parts c and d of Figure 5 show the numbers of changes of HB types 4 and 5 and $\mathrm{HB}$ types 1 and 2, respectively. HB 4 and 5 represent the only connections between water molecules and the channel walls below $245 \mathrm{~K}$. Figure $5 \mathrm{c}$ shows that almost all water molecules are bonded to the tube walls at $40 \mathrm{~K}$. The numbers of HB 4 and HB 5 start to decrease at about 115 and $245 \mathrm{~K}$, respectively. Most of them are broken in the temperature range from 245 to $310 \mathrm{~K}$. In our model, the total number of hydrogen bonds change from 320 to 180 for HB 5 and from 320 to 60 for $\mathrm{HB} 4$. A new hydrogen bond connecting the water hydrogen atom of $\mathrm{HB} 4$ to the oxygen of the terminal $\mathrm{COO}$ group of the dipeptide framework appears at higher temperatures. Its number increases to 60 for the whole MD system at $310 \mathrm{~K}$. These new hydrogen bonds shift their location from the center to the end of the frame. A total of about 400 hydrogen bonds were broken in this temperature range, resulting in a large decrease of the intermolecular van der Waals energy in Figure 4b. HB 1 and 2 in Figure 5d span the walls of the nanotube. Some discontinuities can be observed at $80,115,180,200,245,270,310$, and 370 $\mathrm{K}$ in the slope of both hydrogen bond numbers, and each of them corresponds to a change in the slope of the temperature dependence of the lattice constants. However, all these changes are relatively minor when compared to the dramatic changes in lattice constants as a result of water phase transitions or dipeptide melting. Consequently, the confined water molecules play an important role in the phase transitions of the whole system.

3.1.3. Phases and Mobility of Confined Water. The water molecules enclosed in the peptide nanotube form a subsystem coupled to the peptide via hydrogen bonds. The previous discussion revealed that the water molecules play an important role in the phase transition of the whole system. The richness of phase behavior is well-known for bulk water. The difference between the HB network present in confined water and bulk water suggests that the two systems behave differently.

The radial distribution function (RDF) can provide strong evidence of phase transitions of water molecules. ${ }^{40}$ Our simulation results were used to generate radial distribution functions of $\mathrm{O} \cdots \mathrm{O}$ and $\mathrm{O} \cdots \mathrm{H}$ distances (Figure 6). Figure 6 shows a first peak in $g_{\mathrm{OO}}(r)$ at $2.75-2.8 \AA$, and a first and second peaks in $g_{\mathrm{OH}}(r)$ at 1.85 and $3.05-3.15 \AA$, which is in agreement with those for confined water in the simulations of Engels et al. ${ }^{20}$ From the mean squares displacements of water oxygen atoms and the lattice constants of peptide system, different phases of confined water can be identified in the following temperature ranges.

The well-ordered crystalline phase exists from 40 to $115 \mathrm{~K}$. Experimental observations indicate an ordered array of water molecules in this temperature range. Figure 7a shows a helical structure of water molecules at $40 \mathrm{~K}$. For each water molecule, one hydrogen atom is bonded to the peptide wall by HB 4; each oxygen atom is bonded to both walls by HB 5 and to a hydrogen atom from a nearby water molecule by HB 6 . The remaining hydrogen atom is bonded to an oxygen atom from a neighboring 

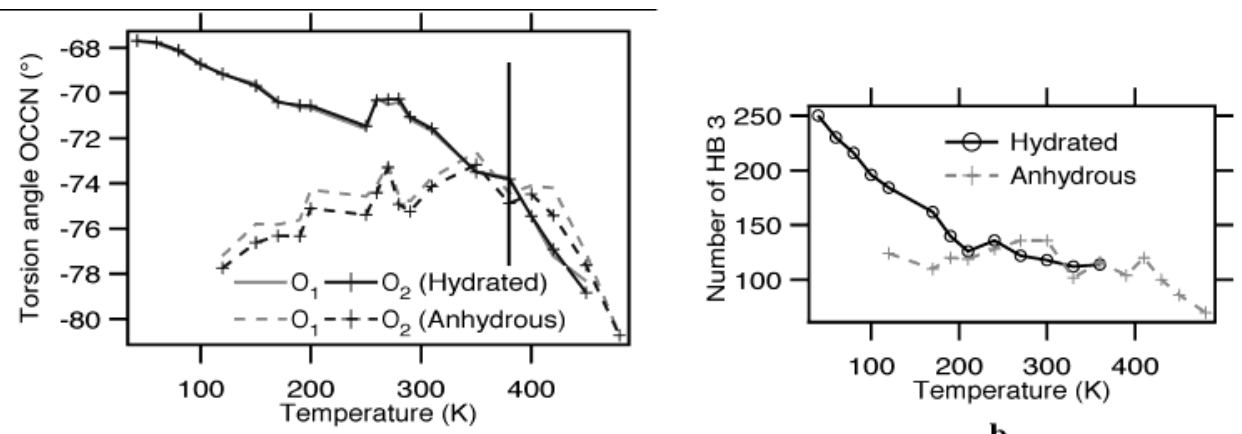

b
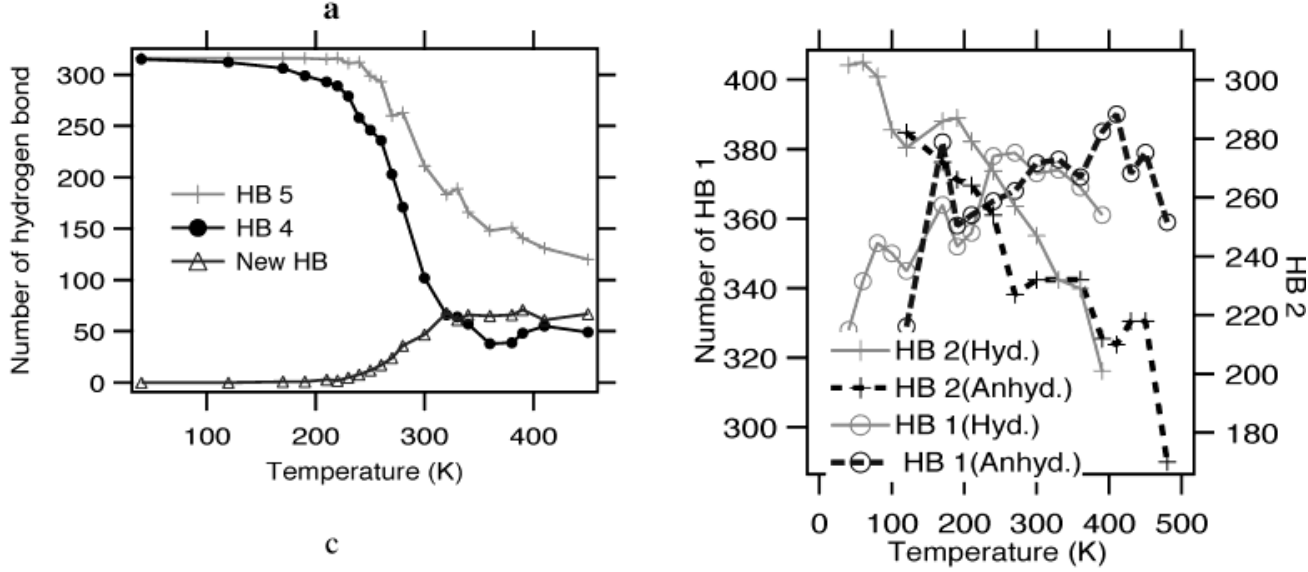

d

Figure 5. Variations of the OCCN torsion angles and numbers of different hydrogen bonds. (a) OCCN torsion angles at the end of the dipeptide framework. (b) Number of hydrogen bonds of type 3. (c) Number of hydrogen bonds between water molecules and the tube wall. (d) Number of hydrogen bonds between dipeptides within each nanotube.

water molecule with HB 6. At 3 kbar, ice-nine (ice IX) shows the same 4-fold helicoidal ordered crystalline structure below $165 \mathrm{~K}^{41}$

An amorphous, disordered phase lacking long-range order exists from 115 to $245 \mathrm{~K}$ (Figure $6 \mathrm{a}, \mathrm{b}^{38}$ ). Differences from the crystalline phase appear on the second and third peaks in the $\mathrm{O} \cdots \mathrm{O}$ RDF along with a merging of the fifth and sixth peaks in the O*m RDF. The origin of this transition is the breaking of $\mathrm{HB} 4$, which is shown in Figure 5c. The loss of this HB 4 releases the corresponding hydrogen atom and allows the related atoms to modify their positions. With the decrease of the number of $\mathrm{HB} 4$, the water molecules increase their disorder. This is the origin of the phase transition of water molecules from the crystalline to the amorphous phase. Experimental results revealed disordered water molecules at $120 \mathrm{~K} .{ }^{11} \mathrm{In}$ the bulk case, the disordered phase of ice-three (ice III) has the same structure as ice-nine with different ordering at $3 \mathrm{kbar}$ and above $165 \mathrm{~K}^{42}$

Above $245 \mathrm{~K}$, a first-order phase transition from amorphous ice to liquidlike phase can be detected. This transition is due to massive breaking of HB 4 and 5, which originate from a sudden increase of the OCCN torsion angle of the peptide system. Some water molecules are disordered in the channel and the helical structure partly evolves after the transition. From ice to liquidlike phase, the value of $g_{\mathrm{OO}}(r) \mathrm{RDF}$ increases suddenly from 3.2 to $3.8 \AA$. Figure $7 c$ illustrates the liquidlike ice structures of water molecules at $260 \mathrm{~K}$. Experiment found that the liquidlike water confined inside carbon nanotubes transforms into a new solid form, i.e., ice nanotube, at $235 \mathrm{~K} .{ }^{43}$ Similar phenomena were also obtained in simulations. ${ }^{23,24}$

In Figure $6 \mathrm{e}$, from 260 to $300 \mathrm{~K}, g_{\mathrm{OO}}(r)$ increases in the range from 3.1 to $3.9 \AA$ and decreases from 4.2 to $4.5 \AA$. This indicates that the water transforms from liquidlike to a complete liquid phase. At $310 \mathrm{~K}$, the water molecules change their mobility. This can be deduced from the MSD in Figure 4a. Figure 6e shows the disappearance of many peaks from 300 to $330 \mathrm{~K}$. Figure $7 \mathrm{~d}$ shows the water configuration at $360 \mathrm{~K}$. The water helix observed at $40 \mathrm{~K}$ is replaced by water clusters. Most water molecules have released their connections to the channel wall. One more phase transition can be found over about $385 \mathrm{~K}$. This phase has been called superheated water. ${ }^{44}$ In this temperature range, the simulated encapsulated water may be quite different from the real confined water.

Our confined water can be treated as quasi-one-dimensional (Q1D) water in the $4.2 \AA$ radius peptide tube. ${ }^{23}$ The selfdiffusion coefficient $(D)$ of water along the tube axis can be calculated from the mean square displacement $\left\langle[R(t)]^{2}\right\rangle$ with $D$ $=\left\langle[R(t)]^{2}\right\rangle /(6 \times t)$. The values are $0.07 \times 10^{-5}, 0.23 \times 10^{-5}$, $0.46 \times 10^{-5}$, and $0.83 \times 10^{-5} \mathrm{~cm}^{2} \mathrm{~s}^{-1}$ for the temperature ranges of $245-275,275-295,295-380$, and $>380 \mathrm{~K}$, respectively. In previous simulations, the self-diffusion coefficients of confined water were $0.44 \times 10^{-5}$ at $298 \mathrm{~K}$ for a $3.4 \AA$ radius peptide tube ${ }^{20}$ and $0.27 \times 10^{-5}$ at $305 \mathrm{~K}$ for $2 \AA$ radius of the gramicidin A channel. ${ }^{45}$ The experimental result is $2.3 \times 10^{-5}$ $\mathrm{cm}^{2} \mathrm{~s}^{-1}$ at $298 \mathrm{~K}$ in bulk water. ${ }^{46}$ Compared to bulk water, the smaller self-diffusion coefficient in the present case is due to HB 4 and 5 and the "new" HB, which limit the water molecule displacement in the channel.

Details about the mobility of the water molecules inside the dipeptide channel can also be obtained by examining the temperature-dependent coordinates of typical water oxygen atoms.

Figure 8 shows the $x, y$, and $z$ coordinates of five water oxygen atoms having different initial $z$ locations. At temperatures below $250 \mathrm{~K}$, the water molecules fluctuate about their equilibrium positions with a small thermal displacement. At 250 $\mathrm{K}$, a few water molecules are slightly displaced. In the 


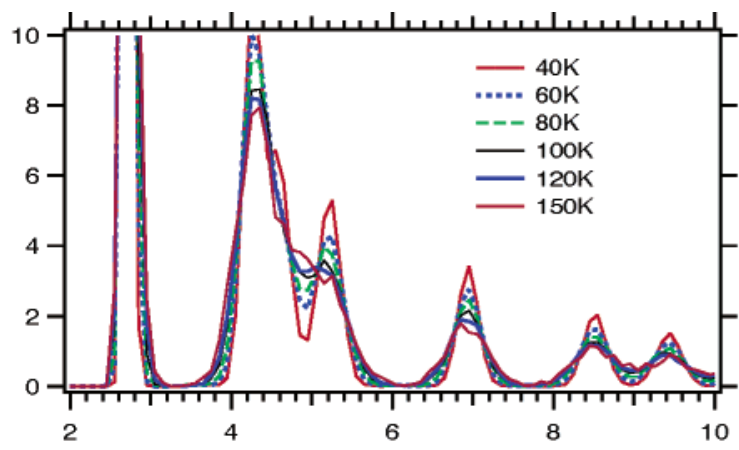

a

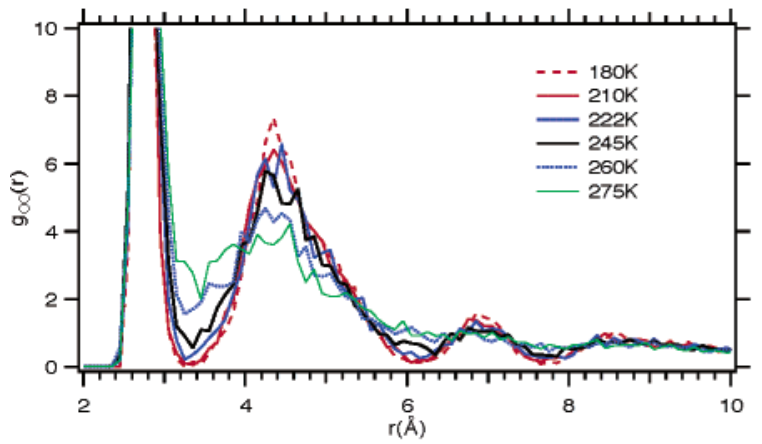

C

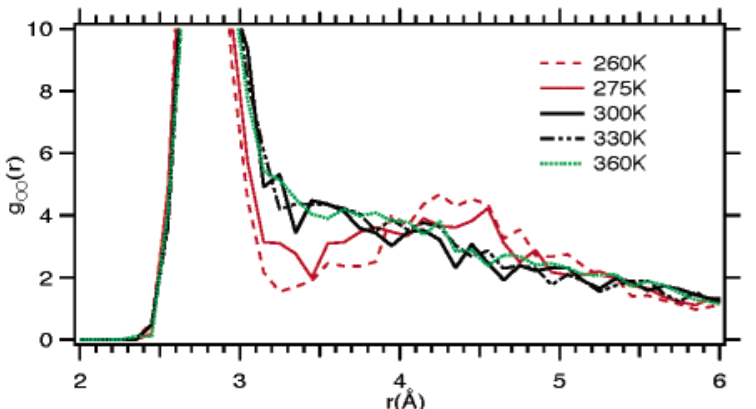

e

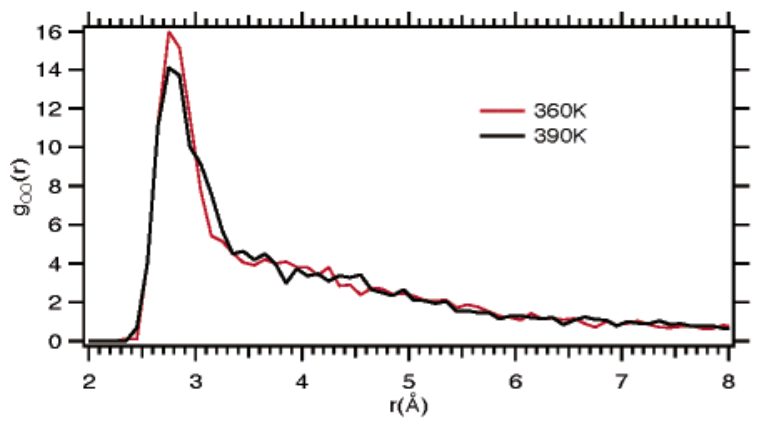

$\mathrm{g}$

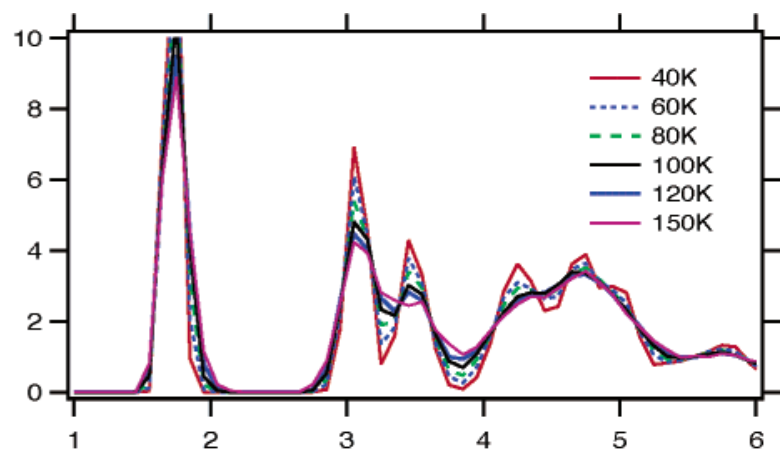

b

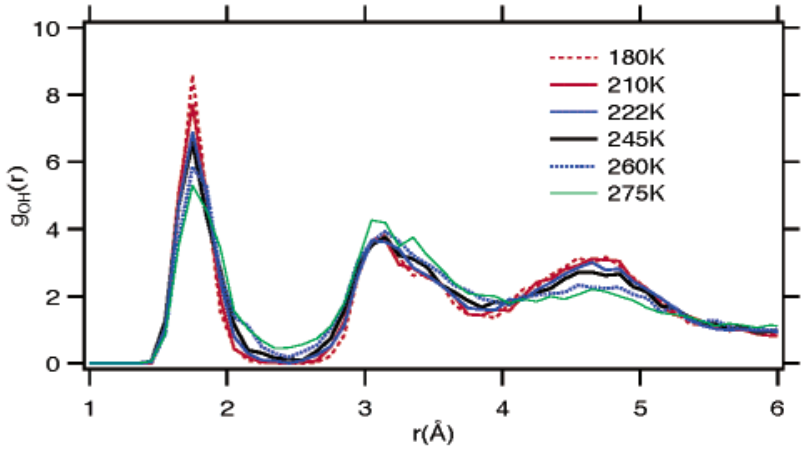

d

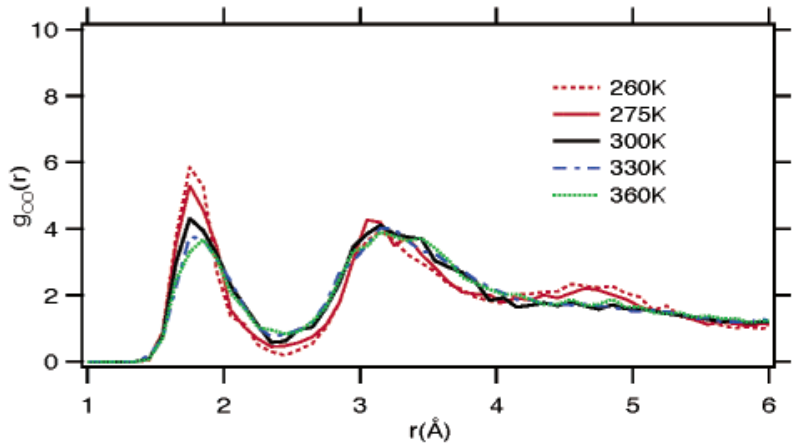

$r(\bar{A})$

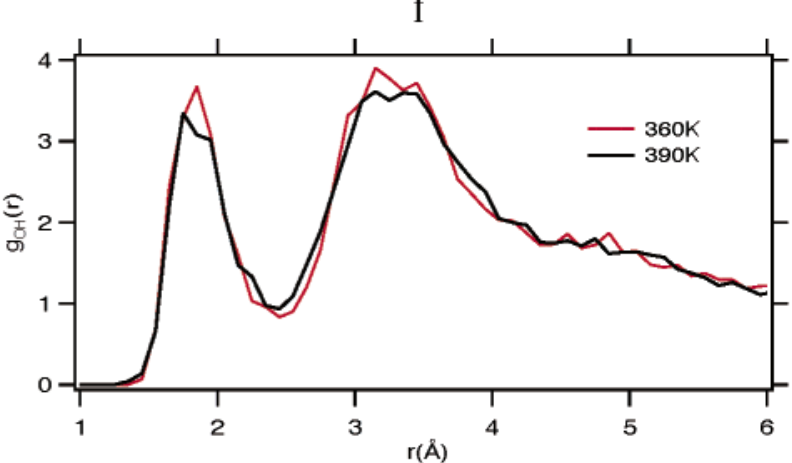

h

Figure 6. The calculated radial distribution functions $g_{\mathrm{OO}}(r)$ and $g_{\mathrm{OH}}(r)$ in different temperature ranges.

temperature range from 250 to $310 \mathrm{~K}$, due to their separation from the wall, some of the water molecules can move freely in the $x y$ plane but only in a limited range along $z$. This is due to the fact that many of them are still bonded to the walls, thus limiting their displacement. Above $310 \mathrm{~K}$, most of the water molecules are separated from the walls and begin to form clusters. The water molecules can thus move freely along the tube. The displacement along $z$ can be as much as $12 \AA$ in the simulation. The flow of water can be observed in Figure 8a. In a finite crystal, the water molecules would be able to leave the channel, thus causing a mass loss of the real system.

A significant mass loss was observed by TGA measurements. The experimental observation which starts at $317 \mathrm{~K}$ is close to our simulation value of $310 \mathrm{~K}$. TGA showed different levels of mass loss that qualitatively agree with the present simulated water mobility level (or self-diffusion coefficient).

3.2. Simulation Results for the Anhydrous Nanotube. The anhydrous nanotube is stable, indicating that the tubular peptide 


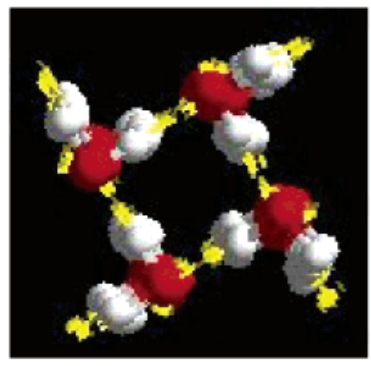

a. $40 \mathrm{~K}$

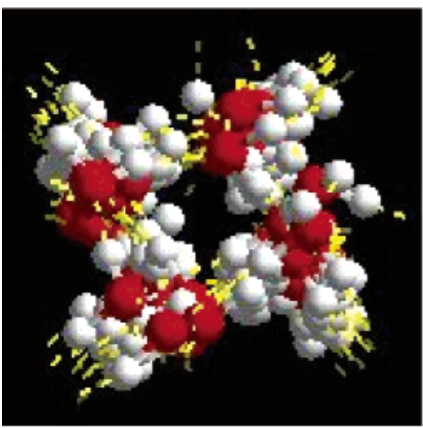

c. $260 \mathrm{~K}$

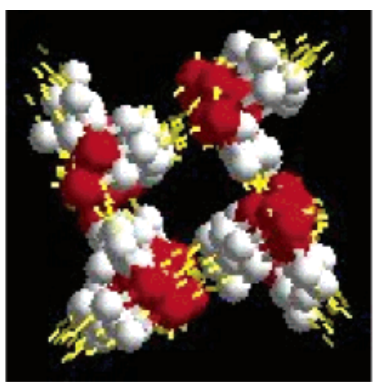

b. $180 \mathrm{~K}$

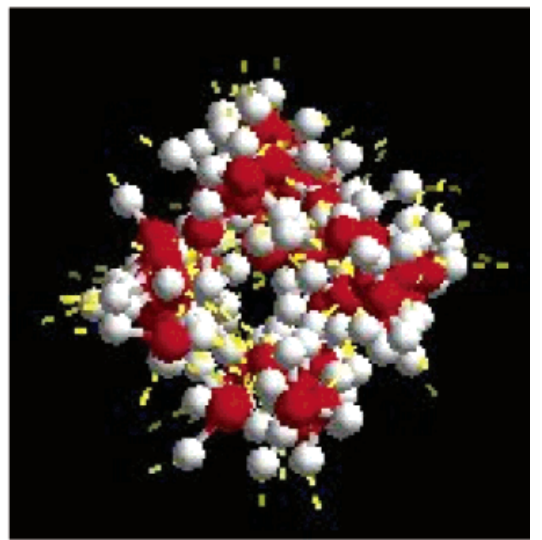

d. $360 \mathrm{~K}$

Figure 7. Structures of the water molecules at different temperatures. (a) Crystalline phase at $40 \mathrm{~K}$. All water molecules are bonded to the peptide nanotube walls forming a well-ordered helix. (b) At $180 \mathrm{~K}$, water molecules form an amorphous and disordered phase. (c) Liquidlike phase at 260 $\mathrm{K}$. Some links with the walls are broken and some water molecules move a short distance. (d) Liquid phase at $360 \mathrm{~K}$. Most water molecules are disconnected from the wall and start to form clusters. They can move a long distance in the tube.

structure might be expected to be observable experimentally. Figure $3 \mathrm{c}, \mathrm{d}$ reveals a dramatic change in the lattice constants at $440 \mathrm{~K}$. Negative thermal expansion of the lattice is not observed in the temperature range from 120 to $310 \mathrm{~K}$. The lattice angles do not deviate from $90^{\circ}$ up to $450 \mathrm{~K}$. Figure 4 a shows the mean square displacement changes at 440 and $470 \mathrm{~K}$. The framework molecules melt at $440 \mathrm{~K}$. Above $435 \mathrm{~K}$ a decomposition of the dipeptide is observed experimentally and the sample changes from white to yellow. Figure $4 \mathrm{~b}$ presents changes of the van der Waals energy of the system and shows clearly a slope change from 420 to $440 \mathrm{~K}$, which in the simulation corresponds to the framework melting. Figure 5a,b,d indicates some transitions inherent in peptide system. We observe some common features at 180, 245, 270, 370, 420, and $440 \mathrm{~K}$ in both hydrated and anhydrous systems. Some of them represent important changes of the whole system, such as 245, 270 , and $440 \mathrm{~K}$. It is interesting to find that the pure peptide system exhibits some characteristic temperatures similar to water at 270 and $370 \mathrm{~K}$.

3.3. Mechanism of NTE. Negative thermal expansion along the tube axis can be observed in both simulations. According to the lattice constant changes in Figure $3 b, d$, the simulations can be divided into two temperature ranges. Below room temperature, only the hydrated simulation reproduces NTE, whereas above room temperature both simulations indicate NTE.

Below room temperature, the confined water is the obvious reason leading to the negative thermal expansion of the $c$ lattice constant. In Figure 5c, changes in HB 4 and 5 connecting water to the peptide provide information on their interactions. Figure 6a,b shows that below $240 \mathrm{~K}$ the distances of the third to sixth nearest oxygen pairs and second and third oxygen-hydrogen pairs decrease. Note that the third peak located between 5.05 and $5.25 \AA$ should be equal to the $c$ lattice constant in the crystalline phase. Figure 6 a shows that the third peak shifts from 5.25 to $5.05 \AA$ when the temperature changes from 40 to 120 $\mathrm{K}$. The systemic lattice constant $c$ changes from 5.219 to 5.206 $\AA$ in the same temperature range. Thus, the confined water contracts much faster than the peptide. As a consequence, the water molecules force the channel wall to contract by means of HB 4 and 5. In this process, the weaker HB 3 disappears, which also contributes to the shortening of the wall along the channel direction. The structural change observed in the peptide (as shown in Figure 5a,c) accelerates the decreasing of $\mathrm{HB} 4$ at about $180 \mathrm{~K}$. The significant structural change of the peptide at $245 \mathrm{~K}$ causes HB 4 and 5 to decrease dramatically. This leads to a release of most of the water molecules from the wall. They partly possess a liquid property that can be identified in Figure $6 \mathrm{c}$,d. The second nearest $\mathrm{O}-\mathrm{O}$ pair shifts from 4.18 to $4.10 \AA$ (Figure 6c), inducing a decrease of the lattice constant $c$ through the wall contact. Below room temperature, the contraction of the confined water is much stronger than the potential expansion tendency of the anhydrous channel, thus leading to the unusual thermal expansion. Figure 5b,c shows that the main contribution of the confined water is over at $310 \mathrm{~K}$ in the channel direction and at $200 \mathrm{~K}$ in $a$ and $b$ directions.

Above room temperature, both simulations reproduced NTE of lattice constant $c$. In the anhydrous case, increases of thermal energy cause weaker intermolecular interactions; HB 3 and 2 decrease at 310 and $350 \mathrm{~K}$ (Figure 5b,d). This leads to the involved oxygen and hydrogen atoms becoming partly free with increases of their mean square displacements (Figure 9). With the reordering of the $\mathrm{HB}$ network around the $\mathrm{COO}$ group, the channel wall formed by the framework retracts in the channel direction. Figure 9 shows the melting of the peptide system with an important increase of the mean square displacements of all atoms at $440 \mathrm{~K}$. The lattice constant $c$ greatly decreases with 


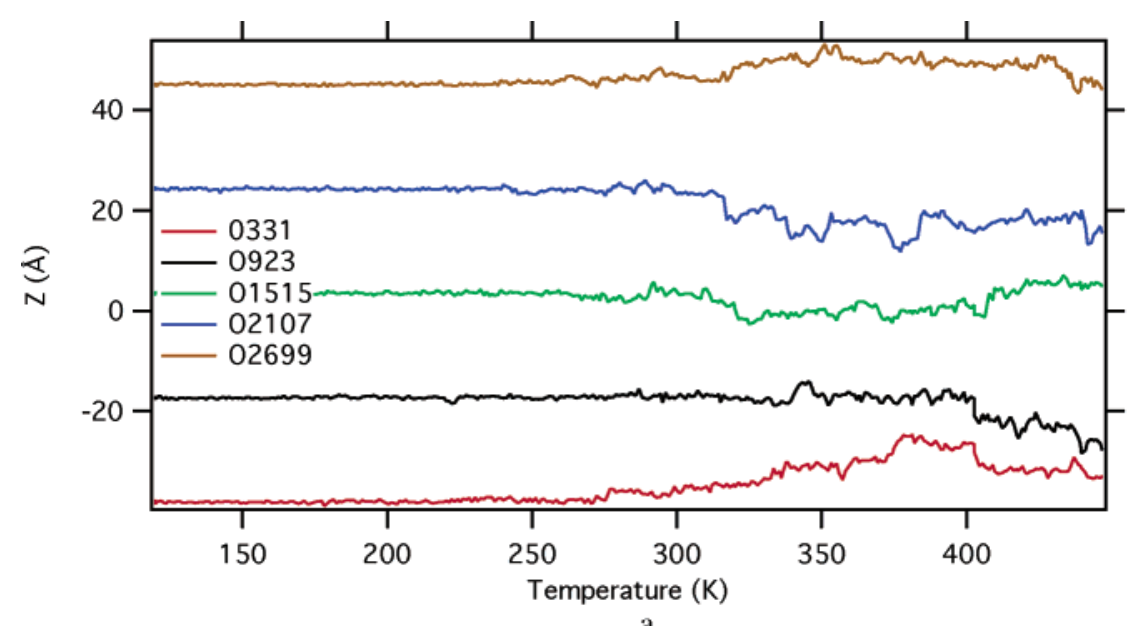

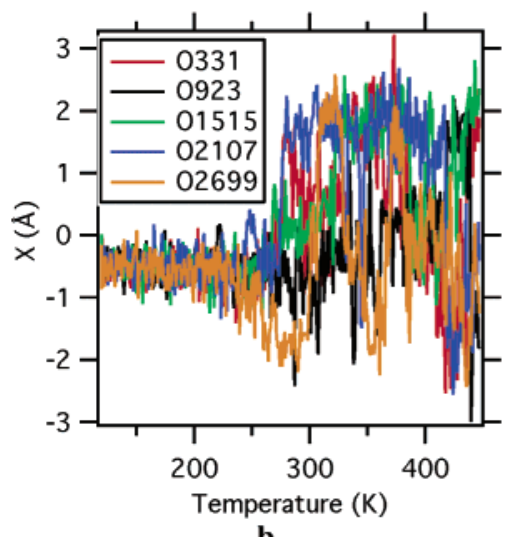

b

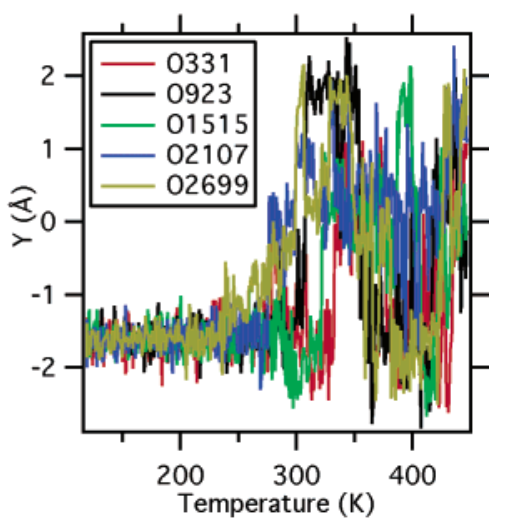

c

Figure 8. Temperature dependence of five oxygen atoms of water molecules from different initial $z$ locations. Coordinates in the (a) $z$ direction, (b) $x$ direction, and (c) $y$ direction. The water molecules are bound to their equilibrium positions with a small thermal displacement at temperatures below $250 \mathrm{~K}$. They can move short distances in the temperature range from 250 to $310 \mathrm{~K}$. Water molecules can move a long distance (>10 $\AA$ ) in the $z$ direction with temperatures higher than $310 \mathrm{~K}$.

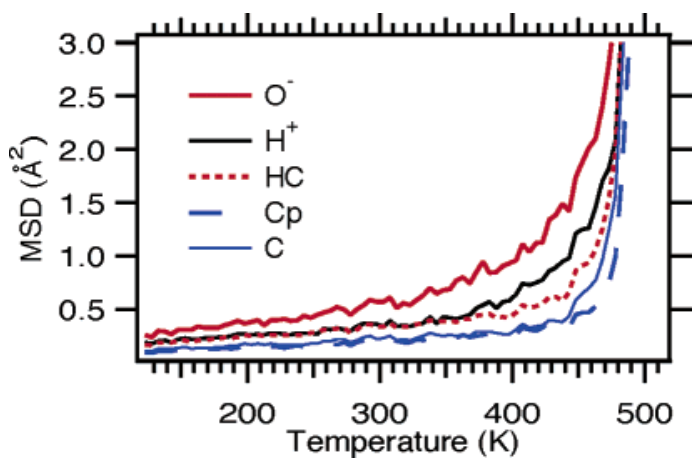

Figure 9. Mean square displacements of different atom types in the anhydrous peptide system. $\mathrm{O}^{-}$belongs to the $\mathrm{COO}$ group, $\mathrm{H}^{+}$to $\mathrm{NH}_{3}$, $\mathrm{HC}$ is the hydrogen connected to carbon, $\mathrm{C}$ is the carbon in the channel wall, and $\mathrm{Cp}$ is the carbon in double rings.

the collapse of the HB network. The mechanism of NTE for the water-containing system is similar to the water-free case above room temperature.

\section{Discussion}

The thermal expansion of the anhydrous nanotube system is positive below room temperature, in contrast to the hydrated system (Figure 3). This indicates that the negative thermal expansion along the tube axis is caused by the water molecules included in the channel. This is in agreement with the model proposed on the basis of experimental observations. ${ }^{11}$ The model arising from these simulations is akin to the situation found in bulk water: the loss of hydrogen bonds leads to a more compact packing of the system. In water, this phenomenon is responsible for the negative volume change when melting. Here the effect is uniaxial due to the nature of the nanotube system.

Water loss is a continuous process from 317 to $420 \mathrm{~K} .{ }^{47}$ After the rupture of the $\mathrm{HB}$ between water and the wall and the increase in the mobility, more and more water molecules escape from the channel. The likelihood is that most water molecules evaporate, whereas a small number of gaslike water molecules still remain inside with a very limited interaction with the tube. Our anhydrous system is just a reasonable model for the real case at higher temperatures.

On the other hand, the use of two simulations on chemically inequivalent systems, i.e., the hydrated containing and anhydrous channel, leads to some questions about the chemical interpretation of our results. The two simulations have a common range when the water leaves the channel at temperatures from 317 to $420 \mathrm{~K}$. For continuity reasons, all the properties of the system in the water-free simulation should be the same as in the first simulation at the common temperature. The second simulation was initiated with a hypothetical model that is identical to the first, but without water molecules. This might theoretically create a matching problem of the two models at the common temperature. Fortunately, after heating from 120 to $370 \mathrm{~K}$, all results of both model calculations were practically identical at temperatures near $370 \mathrm{~K}$. This guarantees that the second simulation can be interpreted as a continuation of the first. It is interesting to note that the model presented here predicts indeed that the anhydrous dipeptide channel is also stable in the crystal 
at temperatures below $373 \mathrm{~K}$. Experiments to confirm this prediction are currently under way.

The refined structure at $120 \mathrm{~K}$ suggests that the HB 4 connects water hydrogen to oxygen in the terminal carboxylate group rather than the keto group, which appears in the MD simulation result at $40 \mathrm{~K}$. The same types of $\mathrm{HB}$ are found experimentally in temperatures above $245 \mathrm{~K}$. The total number of both HBs are almost the same at $310 \mathrm{~K}$, with the total number of them being one-third that below $200 \mathrm{~K}$. The cause of this disparity can be due to many factors. Perhaps the most important of which is the nature of the force field.

The result shows that the confined water changes its mobility at $310 \mathrm{~K}$, which is close to normal human body temperature. If this phenomenon is related to some physiological activity, it is still unclear and awaits further study.

\section{Conclusions}

The structure and phase properties of the tryptophylglycine monohydrate system result from the combined studies of two subsystems. Molecular dynamics simulations were carried out using the ddgmq software and a consistent force field. Two simulations with and without water molecules were performed to model two different temperature ranges. The simulated results agree well with the experiment and provide structural details of the mechanisms of phase transition. Different states of water molecules were observed in the nanotube at different temperature ranges. At temperatures below $115 \mathrm{~K}$, the water molecules exhibit crystalline order and form helical structures. At temperatures between 115 and $245 \mathrm{~K}$, the water molecules are slightly disordered, although a helical structure remains. The liquidlike phase of water exists from 245 to $270 \mathrm{~K}$. At temperatures above $310 \mathrm{~K}$, the water molecules are mobile and form freely moving clusters in the nanotube channel. Below $245 \mathrm{~K}$, the contraction of the crystalline and amorphous water affects the channel wall and causes the negative expansion of the system along the wall direction. The collapse of the solid water $\mathrm{HB}$ and peptide $\mathrm{HB}$ networks leads to the shortening of the channel walls in the temperature range from 245 to $310 \mathrm{~K}$ and above $310 \mathrm{~K}$. This MD simulation not only reproduces well the experimental observations but also gives some insight into the mechanisms of phase transitions and negative thermal expansion.

Acknowledgment. This work was supported by the Swiss National Science Foundation grant number 20-67698.02. We thank the CSCS in Manno and the IT Domain of the EPFL (Lausanne) for the use of the high-performance computing facilities. H.B. thanks the Danish Research Training Council and the Danish Natural Sciences Research Council for additional support.

\section{References and Notes}

(1) Ghadiri, M. R.; Granja, J. R.; Milligan, R. A.; McRee, D. E.; Khazanovich, N. Nature 1993, 366, 324-327.

(2) Zhang, S.; Holmes, T. C.; Lockshin, C.; Rich, A. Proc. Natl Acad. Sci. USA 1993 90, 3334-3338.

(3) Hruby, V. J. Nature Rev. Drug Discovery 2002, 1, 847-858

(4) (a) Bong, D. T.; Clark, T. D.; Granja, J. R.; Ghadiri, M. R. Angew. Chem., Int. Ed. 2001, 40, 988-1011. (b) Hartgerink, J. D.; Clark, T. D.; Ghadiri, M. R. Chem. Eur. J. 1998, 4, 1367-1372. (c) Ghadiri, M. R. Adv Mater. 1995, 7, 675-677.

(5) Fernandez-Lopez, S.; Kim, H.-S.; Choi, E. C.; Delgado, M.; Granja, J. R.; Khasanov, A.; Kraehenbuehl, K.; Long, F.; Weinberger, D. A.; Wilcoxen, K. M.; Ghadiri, M. R. Nature 2001, 412, 452-455.
(6) Wiese, A.; Seydel, U. J. Indust. Microbio. Biotechnol. 1999, 23, 414-424.

(7) Hartgerink, J. D.; Granja, J. R.; Milligan, R. A.; Ghadiri, M. R. J. Am. Chem. Soc. 1996, 118, 8(1), 43-50.

(8) Ranganathan, D.; Lakshmi, C.; Karle, I. L. J. Am. Chem. Soc. 1999, $121,6103-6107$

(9) (a) Görbitz, C. H. Chem. Eur. J. 2001, 7, 5153-5159. (b) Görbitz, C. H. Acta Crystallogr. B 2002, 58, 849-854.

(10) Emge, T. J.; Agrawal, A.; Dalessio, J. P.; Dukovic, G.; Inghrim, J. A.; Janjua, K.; Macaluso, M.; Robertson, L. L.; Stiglic, T. J.; Volovik Y. and Georgiadis, M. M. Acta Crystallogr. 2000, C56, e469-e471.

(11) Birkedal, H.; Schwarzenbach, D.; Pattison, P. Angew. Chem., Int Ed. 2002, 41, 754-756.

(12) Iijima, S. Nature 1991, 354, 56-58.

(13) Partanen, S.; Kaakola, S.; Kaariainen, I. Acta Physiol. Scand. 1979 107, 213-218.

(14) Vellucci, S. V.; Webster, R. A. Eur. J. Pharm. 1981, 76, 255259.

(15) Lobban, C.; Finney, J.; Kuhs, W. J. Chem. Phys. 2000, 112, 7169; Ludwig, R.; Angew. Chem., Int. Ed. 2001, 40, 1808. Klug, D. Nature 2002, 420, 749 .

(16) Mishima, O.; Calvert, L. D.; Whalley, E. Nature 1984, 310, 393.

(17) Mishima, O.; Calvert, L. D.; Whalley, E. Nature 1984, 314, 76

(18) Poole, P. H.; Sciortino, F.; Essmann, U.; Stanley, H. E. Nature 1992, 360, 324

(19) Tanaka, H. Nature 1996, 380, 328.

(20) Engels, M.; Bashford, D.; Ghadiri, M. R. J. Am. Chem. Soc. 1995 117(36), 9151-9158.

(21) Humer, G.; Rasaiah, J. C.; Noworyta, J. P. Nature 2001, 414, 188 190.

(22) Maniwa, Y.; Kataura, H.; ABE, M.; Suzuki, S.; Achiba, Y.; Kira, H.; Matsuda, K. J. Phys. Soc. Jpn. 2002, 71, 2863.

(23) Koga, K.; Gao, G. T.; Tanaka, H.; Zeng, X. C. Phys. A 2002, 314, 462.

(24) Koga, K.; Gao, G. T.; Tanaka, H.; Zeng, X. C. Nature 2001, 412 , 802; and Koga, K.; Zeng, X. C.; Tanaka, H. Phys. Rev. Lett. 1997, 79, 5262 .

(25) Dujardin, E.; Ebbesen, T. W.; Hiure, H.; Tanigaki, K. Science 1994, $265,1850$.

(26) Koga, K.; Parra, R. D.; Tanaka, H.; Zeng, X. C. J. Chem. Phys 2000, 113, 5037.

(27) Pan, Y.; Brown, D.; Chapuis, G. Mol. Simulation 2003, 29, 509518.

(28) Pan, Y.; Brown, D.; Chapuis, G. Phys. Rev. B, 2002, 65(18), $184205 / 1-8$.

(29) Brown, D.; Minoux, H.; Maigret, B. Comput. Phys. Commun. 1997 103, 170-186. Brown, D.; Maigret, B. Speedup, Proceedings of the 24th SPEEDUP Workshop, Berne Sept 24-25, 1998, Vol. 12(2), pp 33-41. See also The gma User Manual Version 3, available at http:// www.univ-savoie.fr/labos/lmops/brown/gmq.html.

(30) Hammonds, K. D.; Ryckaert, J.-P. Comput. Phys. Commun. 1991, $62,336-351$

(31) Hwang, M.-J.; Stockfischand, T. P.; Hagler, A. T. J. Am. Chem. Soc. 1994, 116, 2515-2525.

(32) Hagler, A. T.; Dauber, P.; Lifson, S. J. Am. Chem. Soc. 1979, 101 5122-5130 and 5131-5141.

(33) Cerius2, Molecular Simulations, San Diego, CA, 1997.

(34) Rappé, A. K.; Goddard, W. A. J. Phys. Chem. 1991, 95, 33583363.

(35) Ewald, P. P. Ann. Phys. 1921, 64, 253.

(36) Smith, W. Comput. Phys. Commun. 1992, 67, 392-406.

(37) Smith, W. CCP5 Newslett. 1986, 21, 37.

(38) Matsumoto, M.; Salto, S.; Ohmine, I. Nature 2002, 416, 409-413, and Koga, K.; Tanaka, H.; Zeng, X. C. Nature 2000, 408, 564-567.

(39) Gao, G. T.; Zeng, X. C.; Tanaka, H. J. Chem. Phys. 2000, 113, 5037.

(40) Ludwig, R. Angew. Chem., Int. Ed. 2001, 40, 1808-1827.

(41) Placa, S. J.; Hamilton, W. C.; Kamb, B.; Prakash, A. J. Chem. Phys. 1973 58, 567-580.

(42) Kamb, B.; Prakash, A. Acta Crystallogr. 1968 B24, 1317-1327.

(43) Maniwa, Y.; Kataura, H.; Abe, M.; Suzuki, S.; Achiba, Y.; Kira,

H.; Matsuda, K. J. Phys. Soc. Jpn. 2002, 71, 2863-2866.

(44) Mishima, O.; Stanley, H. E. Nature 1998, 396, 329.

(45) Chiu, S.; Subramaniam, S.; Jakobsson, E. Biophys. J. 1999, 76 1939-1950.

(46) Price, W. S.; Ide, H.; Arata, Y. J. Phys. Chem. A 1999, 103, 448450; also Mills, R. J. Phys. Chem. 1973, 77, 685-688.

(47) Birkedal, H. Ph.D. thesis, Institute of crystallography, University of Lausanne, Switzerland, 2000.

(48) For an update on crystallographically determined peptide structures, see: Görbitz, C. H. Curr. Opin. Solid State Mater. 2002, 6, 109-116. 